Reprod. Nutr. Dévelop., 1986, 26 (1 B), 371-372.

\title{
Variations nycthémérales de la lipémie et de la glycémie au niveau des voies afférentes et efférentes du foie chez le veau préruminant
}

D. DURAND, D. BAUCHART

Laboratoire d'Etude du Métabolisme énergétique I.N.R.A., Theix 63122 Ceyrat, France.

L'étude du métabolisme hépatique des bovins par la mesure directe in vivo des flux des métabolites captés et émis par le foie a été réalisée au cours de la journée par Baird, Symonds et Ash (1975) et Lomax et Baird (1983) chez la Vache laitière, et par Durand, Bauchart et Lefaivre (1984) chez le Veau préruminant. Les objectifs de ce travail étaient d'apprécier les variations nycthémérales des concentrations des sangs afférents et efférents au foie en glucose (Glu), triglycérides (TG), acides gras non estérifiés (AGNE), cholestérol libre (CL) et esters de cholestérol (EC).
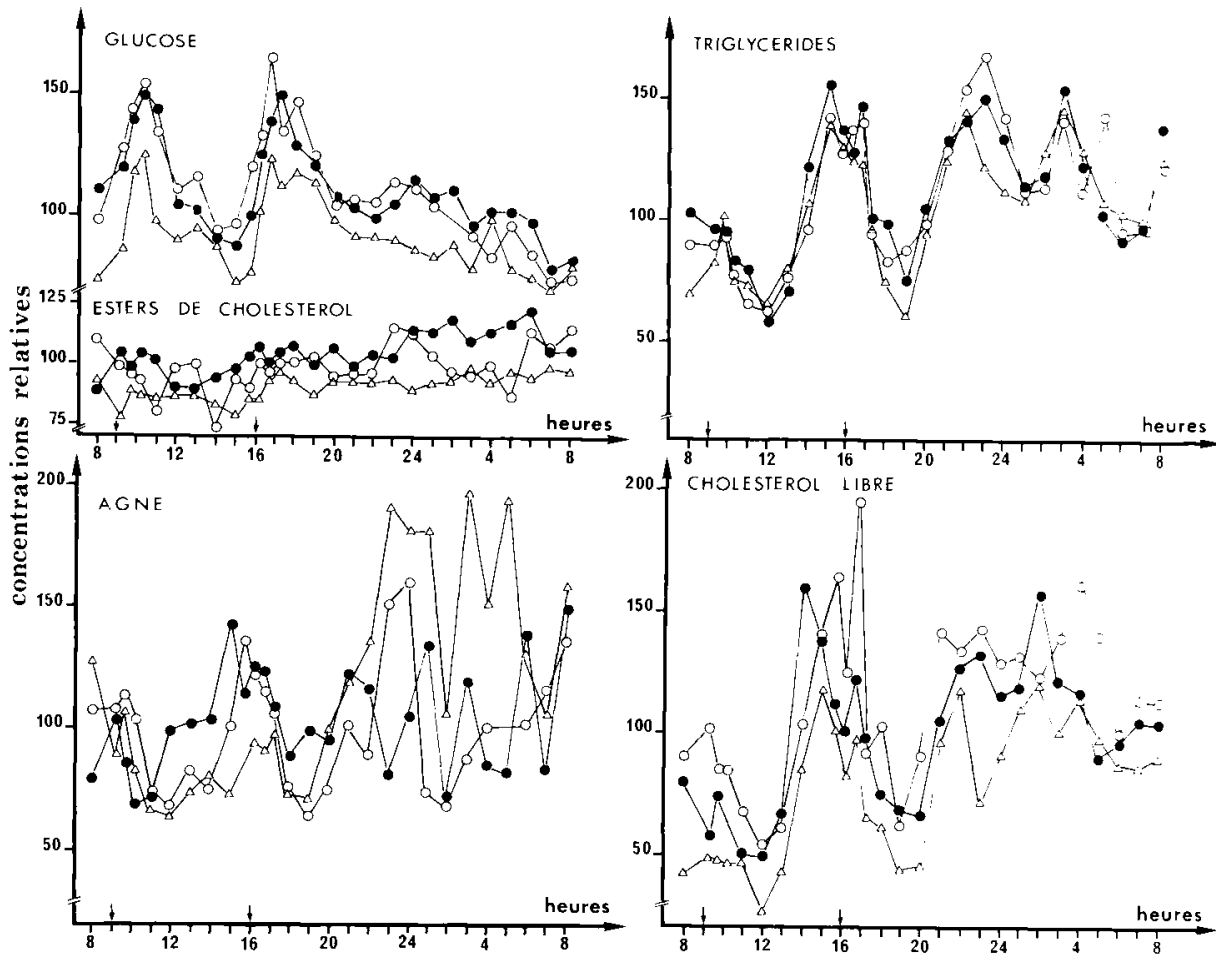

FIG. 1. - Variations nycthémérales des concentrations relatives en G/u, TG, AGNE, CL et EC dans la veine porte $(\bullet)$, la veine sus-hépatique $(O)$ et l'artère hépatique $(\triangle)$. Les valeurs de références $(100 \%)$ correspondent à la moyenne des concentrations dans les trois vaisseaux pour l'ensemble des prélèvements soit : Glu : $130 \mathrm{mg} / 100 \mathrm{ml}$; TG : $58,4 \mathrm{mg} / 100 \mathrm{ml}$; AGNE : 10,2 $\mathrm{mg} / 100 \mathrm{ml}$; CL : $8,5 \mathrm{mg} / 100 \mathrm{ml}$; EC : $100,2 \mathrm{mg} / 100 \mathrm{ml}$. (1) prise du repas.

Reproduction, Nutrition, Développement, $n^{\circ} 1$ B-86. -17 
Matériel et méthodes. Cette étude a été réalisée chez 4 veaux mâles préruminants Pie noir âgés de 4 semaines $(46,5 \pm 3,7 \mathrm{~kg})$ recevant en deux repas égaux $(9 \mathrm{~h}$ et $16 \mathrm{~h}$ ) un lait de remplacement (suif : $23 \% \mathrm{MS}$ ) à raison de $1,7 \% \mathrm{du}$ poids vif. Les prélèvements sanguins (28, fig. 1) ont été effectués à l'aide de cathéters implantés en chronique dans l'artère hépatique, la veine porte et une veine sus-hépatique. Les concentrations plasmatiques ont été déterminées par voie enzymatique (TG, EC, CL et GLu) et par titrimétrie (AGNE).

Résultats et discussion. Après les deux pics postprandiaux, la glycémie baisse notamment au cours de la nuit ; les faibles valeurs observées au niveau du sang sus-hépatique indiqueraient une phase de captation de glucose par le foie en période nocturne. La similitude d'évolution de la triglycéridémie dans les trois vaisseaux montre que le foie du Veau capte apparemment autant de TG qu'il en émet. Les fortes triglycéridémies observées $7 \mathrm{~h}$ après les repas correspondent à l'arrivée de chylomicrons riches en TG alimentaires, ce que confirme l'absence du second pic lorsque l'animal ne reçoit que le repas du matin (Durand et Bauchart, non publié). La présence de pics à 3 et $8 \mathrm{~h}$ peut s'expliquer par l'arrivée de salves supplémentaires de TG alimentaires et (ou) par la synthèse de triglycérides endogènes par l'intestin consécutive à une forte captation d'AGNE au cours de la nuit. Les baisses des teneurs en AGNE, maximales $2 \mathrm{~h}$ après les repas, pourraient correspondre à une inhibition partielle de la lipolyse tissulaire en période d'hyperinsulinémie. Leur élévation plus précoce ( $3 \mathrm{~h}$ après les repas) au niveau porte proviendrait d'une mobilisation du tissu adipeux mésentérique, antérieure à celle des autres tissus. Au cours de la nuit, à une forte mobilisation des réserves corporelles observée au niveau artériel, correspond une captation intense d'AGNE par l'intestin et le foie.

La concentration du sang porte en $C L$ et en $E C$, supérieure à la concentration artérielle pendant tout le nycthémère, indique qu'une fraction non négligeable du cholestérol absorbé ou synthétisé par l'intestin est émise par la voie porte vraisemblablement sous forme de HDL naissantes. Excepté à 14, 17, 19 et $2 \mathrm{~h}$, le foie enrichit le sang sus-hépatique en $C L$, par contre il semble capter activement les EC notamment au cours des périodes $14-18 \mathrm{~h}$ et $1-6 \mathrm{~h}$.

Chez le Veau préruminant soumis à un niveau d'alimentation modéré (croît de $428 \pm 71 \mathrm{~g} / \mathrm{j}$ ), la captation et la production de TG par le foie semblent s'équilibrer. En revanche, le foie et principalement l'intestin captent activement les AGNE au cours de la nuit. L'intestin enrichit généralement le sang porte en $C L$ et en $E C$ alors que le foie capte des $E C$ et émet du CL pratiquement tout le nycthémère.

Baird G. D., Symonds H. N., Ash R., 1975. Some observations on metabolite production and utilization in vivo by the gut and liver of adult dairy cows. J. agric. Sci., Camb., 85, 281-296.

Durand D., Bauchart D., Lefaivre J., 1984. In vivo hepatic balance of lipids and glucose in the calf ; effect of sorbitol intake. Can. J. anim. Sci., 64 (suppl.), 238-239.

Lomax M. A., Baird G. D., 1983. Blood flow and nutrient exchange across the liver and gut of the dairy cow. Br. J. Nutr., 49, 481-496. 